\title{
Evaluación del consumo de sustancias tóxicas entre los estudiantes de Ciencias de la Salud iraníes
}

\author{
Jamshid Ahmadi, MD, Prof. de Psiquiatría \\ Ali Javadpour, MD, Médico Residente en \\ Psiquiatría \\ Hafez Hospital, Shiraz \\ IRÁN
}

\begin{abstract}
RESUMEN - Objetivos: Las estrictas medidas que se han utilizado para prohibir la venta y el consumo de sustancias tóxicas legales no ha modificado la tendencia al alza en el consumo de sustancias tóxicas en Irán. El objetivo de este estudio es el de conocer el consumo de sustancias tóxicas entre los estudiantes de Ciencias de la Salud iraníes.

Participantes: Se eligió a 346 estudiantes al azar, donde el 43,9\% eran mujeres y el $56,1 \%$, hombres.

Método: Se distribuyó un cuestionario confidencial que fue cumplimentado por los estudiantes y recogido posteriormente.

Hallazgos: De todos los participantes, el 34,7\% admitieron haber consumido alguna sustancia tóxica:Los cigarrillos (el 28\%), son las que se consumían con mayor frecuencia. El resto de las sustancias fueron: alcohol (el 15\%), opio (el 8,4\%), cannabis (el 1,2\%), heroína (el 0,3\%) y LSD (el 0,3\%). Algunos consumían más de una de estas drogas.

Conclusiones: El consumo de sustancias tóxicas estaba significativamente ligado al sexo (el $50 \%$ de los hombres y el 15,2\% de las mujeres consumieron en algún momento alguna sustancia tóxica y el 11,3\% de los hombres y el 1,4\% de las mujeres eran consumidores habituales). El motivo más común era el de obtener placer tanto para antiguos consumidores como para los actuales.
\end{abstract}

\section{Introducción}

Las drogodependencias pueden reducir el rendimiento académico de los estudiantes; por lo tanto, es necesario que la Universidad evalúe el alcance del problema, sepa cuáles son los factores que contribuyen a su existencia, reconozca los signos y los síntomas y lleve a cabo intervenciones educativas con el fin de identificar y prevenir la dependencia que puedan provocar estas sustancias (Coleman, et al. 1997).

En un estudio que evaluaba el abuso de distintas drogas entre los estudiantes de Ciencias de la Salud, se descubrió que el $10 \%$ de las enfermeras eran adictas y que, 
para muchas, el problema había comenzado en la Universidad (Coleman, et al. 1997). No se sabe mucho acerca de las drogodependencias en Irán. En lo que a las drogas respecta, es importante mencionar que se pueden producir arrestos y encarcelaciones por posesión y consumo de sustancias ilegales tales como los narcóticos/opiáceos, cannabis, cocaina, LSD, estimulantes y alucinógenos. Por otro lado, el tabaco no está prohibido ni por motivos legales ni religiosos.

En otro estudio que valoraba la proporción de drogodependientes entre los estudiantes universitarios iraníes, el 24\% afirmó haber consumido sustancias tóxicas en algún momento de su vida mientras que el $75 \%$ aseguró que nunca las habían probado. La sustancia con mayores consumos era el hachís (no se incluyó al tabaco). Si el $90 \%$ de los participantes manifestaron que preferían fumar opio, para el 5\% lo mejor era inyectárselo. La mayoría (el 99\%) consumía sustancias tóxicas en las fiestas que celebraban con sus amigos. Se descubrió que la proporción de consumo de sustancias tóxicas era mayor en los hombres que en las mujeres (Merchant, Pournadeali, Zimmer, Ronaghy 1976).

Hay que tener en cuenta que la mayoría de la población iraní (alrededor del 60\%) tienen menos de 25 años y un gran número de ellos son estudiantes de enseñanza secundaria y universitaria.

En un estudio que evaluaba la proporción del consumo de sustancias tóxicas entre los estudiantes mayores de enseñanza secundaria, se descubrió que el $14 \%$ de los chicos afirmaban que fumaban cigarrillos y el $5,7 \%$ consumían otro tipo de sustancias tóxicas (Moosavi 2000).

Teniendo en cuenta el hecho de que los estudiantes de Ciencias de la Salud, formaran parte en un futuro del personal médico y que tendrán una relación más directa y frecuente con los pacientes, pueden influir en dichos enfermos con su comportamiento y actitudes, por lo que es importante determinar la proporción de consumo de sustancias tóxicas entre ellos.

\section{Material y método}

Muestra: Seleccionamos al azar a 473 estudiantes de diferentes Facultades pertenecientes a la Universidad de Ciencias de la Salud en Shiraz, durante el año 2000. Respondieron 346 (la proporción de respuestas fue del $73 \%$ ).

Procedimiento: Se distribuyó un cuestionario de elección múltiple basado en un estudio anterior (Mckay, A.J. Hawthorne, V.M. Mc Cartney, H.N. 1973) que fue completado por los estudiantes y entregado al final de la sesión. Tras ser distribuido, se explicaron las razones por las que se realizaba el estudio y se informó a los estudiantes que sus respuestas serían confidenciales. Los participantes contaron con 10 minutos para completar y devolver el cuestionario que incluía preguntas sobre la edad, el sexo, el estado civil y si tomaban o no algún fármaco psicoactivo, sobre todo sustancias ilegales (alcohol, opio, heroína, cannabis, y otras sustancias psicotropas) tanto ahora como en el pasado (al hablar del momento actual nos referimos a un consumo habitual durante al menos los últimos 30 días). Mediante esta investigación también se pretendía identificar las causas, tales como el hábito, la dependencia (con el fin de evitar síntomas tras el abandono), el placer, la tensión, la depresión y otras responsables del consumo de sustancias tóxicas. 
Tabla I

Sujetos que afirmaron haber consumido sustancias de abuso en el pasado o ahora

\begin{tabular}{lcccc} 
& \multicolumn{2}{c}{ En alguna ocasión } & \multicolumn{2}{c}{ Consumidores habituales } \\
\cline { 2 - 5 } Sustancia & Número & $\%$ & Número & $\%$ \\
\hline Alcohol & 52 & 15 & 6 & 1.7 \\
Tabaco & 97 & 28 & 19 & 5.5 \\
Opio & 29 & 8.4 & 5 & 1.4 \\
Heroína & 3 & 0.9 & 1 & 0.3 \\
Psicodélicos & 2 & 0.6 & 1 & 0.3 \\
Cocaína & 0 & 0 & 0 & 0 \\
Hashish & 9 & 2.6 & 1 & 0.3 \\
Marijuana & 7 & 2 & 3 & 0.9 \\
Morfina & 0 & 0 & 0 & 0 \\
\hline A & & 0 &
\end{tabular}

A los sujetos se les permitió señalar más de una sustancia.

Análisis: El análisis de los datos se llevó a cabo mediante un programa SPSS, empleándose además el test chi-cuadrado para calcular las frecuencias y la t de Student para comparar los distintos instrumentos.

Hallazgos: Del total de los participantes, $152(43,9 \%)$ eran mujeres y $194(56,1 \%)$ hombres. La tabla I muestra el consumo de cada una de las sustancias. Se observa que existe un consumo importante de tabaco (el $28 \%$ habían fumado en alguna ocasión y el $5,5 \%$ eran fumadores habituales). El consumo de sustancias tóxicas se relacionó con el sexo de manera significativa ( $\mathrm{p}<0,001)$. El $50 \%$ de los hombres y el $15,2 \%$ de las mujeres afirmaron haber consumido sustancias tóxicas en algún momento de sus vidas, y además, el 11,3\% de los hombres y el 1,4\% de las mujeres eran consumidores habituales. Existía una relación negativa entre los ingresos familiares y el consumo de sustancias tóxicas (el 10,8\% de las personas con renta baja frente al 5,6\% de personas de renta alta eran consumidores habituales). Existía una relación positiva entre el año de la carrera y el consumo de estas sustancias de abuso (el 3,4\% de los jóvenes frente al $10,7 \%$ de los mayores eran consumidores habituales. El 5,9\% de los estudiantes solteros y el $10,5 \%$ de los casados también consumían habitualmente.

Las razones que adujeron aquellos estudiantes que habían consumido sustancias tóxicas en algún momento de su vida fueron las siguientes: La búsqueda de placer (el $61,7 \%$ ), la imitación social (el 4,2\%), la tensión (el 10,8\%) y la depresión (el 6,7\%). Las razones de los consumidores habituales fueron la búsqueda de placer (el 62,5\%), el hábito (el 29,2\%), la tensión (el 16,7\%), la necesidad (el 12,5\%) y la imitación social (el 4,2\%). El 12,5\% aportó otras razones (algunos estudiantes aportaron más de una razón).

Discusión: Se descubrió que la nicotina era la sustancia más consumida por los estudiantes iraníes de Ciencias de la Salud mientras que los occidentales preferían el alcohol (Kory and Crandall 1984). No se ofreció ninguna información sobre el consumo de cocaína, siendo importante señalar el hecho de que en Irán es muy difícil obtener cocaína, LSD u otras sustancias psicodélicas. Tan solo el $11,3 \%$ de los hombres y el $1,4 \%$ de las mujeres eran consumidores 
habituales. Hay que tener en cuenta que el hecho de que una persona admita públicamente que consume sustancias tóxicas (sin incluir el consumo de los cigarrillos) supone su expulsión de la Universidad, además la baja tasa de prevalencia de usuarios podría ser debida a la posibilidad de que no se hubiera informado sobre ellos. Se descubrió que la búsqueda de placer fue la causa principal que indujo al consumo de sustancias tóxicas tanto entre los consumidores anteriores como en los actuales. También se observó que se consumían importantes can tidades de opio. En Irán existe una antigua tradición según la cual el opio se utilizaba para tratar enfermedades mentales, además de su empleo con objeto de buscar placer y con fines sociales.

Se descubrió que el consumo de sustancias tóxicas estaba significativamente relacionado con el sexo, relación que se acusaba más entre los hombres que entre las mujeres, lo que indica que la sociedad iraní es más permisiva con los hombres. Las sustancias tóxicas que más se habían consumido y que actualmente se consumían eran por este orden los cigarrillos, el alcohol y el opio. La causa que indujo al consumo de sustancias tóxicas tanto a los antiguos consumidores como a los actuales fue la búsqueda de placer.

\section{Bibliografía}

COLEMAN, E.A., HONEYCUTT, G., OGDEN, B., MCMILLAN, D.E., O'SULLIVAN, P.S., LIGHT, K., WING FIELD, W. Assessing substance abuse among health care students and the efficacy of educational interventions. Journal of Prof-Nurse, 13 (1) 28-37, 1997.

KORY, W.P.,CRANDELL,L.A. "Non medical drug use patterns among medical students". The International Jour nal of Addiction 19 (8) 871-884, 1984.

MADDUX, J.F., HOPPE, S.K., GOSTELLO, R.M. Psychoactive Substance Use Among Medical students American Journal of Psychiatry 143: 187-191, 1986.

MCKAY, A.J., HAWTHORNE, V.M., MCCARTNEY, H.N. "Drug Taking Among Medical Students at Glasgow University “ British Medical Journal, 1, 540-543, 1973.

MERCHANT, N.M., POURNADEALI, E., ZIMMER, S.P., RONAGHY, H.A. "Factors related to drug abuse among Iranian university students". Pahlavi Medical Jour nal 7(4)516-528, 1976.

MOOSAVI, GH. "Substance use among Iranian high school students" presented at fourth Iranian Seminar on Children and Adolescents Mental Health,Zanjan,Iran,1820 October, 2000.

Dirección para correspondencia:

Jamshid Ahamdi, MD

P.O. Box 71345-1416

Hafez Hospital

Shiraz

IRÁN 